\title{
Candida dubliniensis Isolated from the Sputum of a Patient with End-stage Liver Cirrhosis
}

\author{
Ryosuke Tsuruta ${ }^{1}$, Yasutaka Oda ${ }^{1}$, Hidekazu Mizuno ${ }^{2}$, Hirotaka Hamada ${ }^{1}$, \\ Takashi Nakahara ${ }^{1}$, Shunji Kasaoka ${ }^{1}$ and Tsuyoshi Maekawa ${ }^{1}$
}

\begin{abstract}
Candida dubliniensis is rare and very similar to C. albicans. To date, detailed clinical reports on $C$. dubuliniensis recovered from an immunocompromised patient have not been described in Japan. A 71-year-old man with end-stage liver cirrhosis had been treated for suppurative omarthritis due to methicillin-resistant Staphylococcus aureus (MRSA). Anti-MRSA agents and broad-spectrum antimicrobials but no antifungal agents had been administrated. C. dubliniensis, isolated from the sputum, was eliminated by selective digestive decontamination and supportive therapy. This case emphasizes the need to recognize this emerging Candida sp., C. dubliniensis in cases of opportunistic infection.
\end{abstract}

Key words: susceptibility, micafungin, methicillin-resistant Staphylococcus aureus, selective digestive decontamination

(DOI: 10.2169/internalmedicine.46.6285)

\section{Introduction}

Candida albicans has been the predominant etiologic agent of candidiasis. The incidence of candidiasis caused by other species of Candida, including C. glabrata, C. krusei and $C$. tropicalis, has increased (1). However, $C$. dubliniensis has not been known in Japan because of the difficulty in discriminating it from C. albicans. Although it is said that C. dubliniensis colonies appear dark green on the commercially available chromogenic agar medium (CHROMagar ${ }^{\mathrm{TM}}$, Paris, France) in contrast to light blue-green colonies of $C$. albicans, it is not easily distinguishable in practice. Therefore, $C$. dubliniensis has possibly been misidentified as $C$. albicans $(2,3)$. If so, it is suspected that some $C$. albicans isolates resistant to fluconazole (FLCZ) may involve C. $d u b$ liniensis isolates because of their ability to rapidly develop FLCZ resistance in vitro (4). Furthermore, there is a controversy of which antifungal agents are necessary for treatment of candidiasis due to $C$. dubliniensis because the patients have multiple underlying conditions such as human immunodeficiency virus (HIV)-infected and with end-stage liver disease. As far as we know, this is the first Japanese case report demonstrating the process to yield the identification as
C. dubliniensis from the clinical specimen.

\section{Case Report}

A 71-year-old man was transferred to our ICU because of respiratory failure. He was diagnosed as having alcoholic liver injury and left suppurative omarthritis, which developed 5 days after artificial head replacement for humeral comminuted fracture. The curettage and drainage were performed for suppurative omarthritis at the previous hospital. Methicillin-resistant Staphylococcus aureus (MRSA), Pseudomonas aeruginosa and Klebsiella pneumoniae had been isolated from the pus on the wound and $200 \mathrm{mg}$ per day of arbekacin (ABK) and $600 \mathrm{mg}$ per day of ciprofloxacin (CPFX) had been administered.

On admission, he appeared restless. His heart rate was 80 beats/min, respiratory rate was 24 breaths/min, blood pressure was $175 / 90 \mathrm{mmHg}$, and body temperature was $37.6^{\circ} \mathrm{C}$. His breath sounds were weak bilaterally. There were no abnormal findings on his heart, abdomen, or nervous system but epidermolysis and swelling on his left shoulder were seen. His laboratory data on admission are shown in Table 1 . These data showed hypoalbuminemia, liver dysfunction and impaired oxygenation. White-cell count, C-reactive

${ }^{1}$ Advanced Medical Emergency and Critical Care Center, Ube and ${ }^{2}$ Division of Laboratory, Yamaguchi University Hospital, Ube Received for publication October 4, 2006; Accepted for publication January 17, 2007

Correspondence to Dr. Ryosuke Tsuruta, ryosan-ygc@umin.ac.jp 
Table 1. Laboratory Data on Admission

\begin{tabular}{|c|c|c|c|c|c|c|c|c|}
\hline [Peripheral blood] & & & [Biochemistry] & & & [Coagulation] & & \\
\hline White-cell count & 13,740 & $/ \mu 1$ & Urea nitrogen & 9 & $\mathrm{mg} / \mathrm{dl}$ & Prothrombin time & 15.4 & $\operatorname{secs}$ \\
\hline Stab neutrophils & 6 & $\%$ & Creatinine & 0.6 & $\mathrm{mg} / \mathrm{dl}$ & Control & 12.1 & $\operatorname{secs}$ \\
\hline Segmented neutrophils & 84.5 & $\%$ & C-reactive protein & 7.63 & $\mathrm{mg} / \mathrm{dl}$ & Partial thromboplastin time & 41.2 & secs \\
\hline Eosinophils & 0.5 & $\%$ & Glucose & 146 & $\mathrm{mg} / \mathrm{dl}$ & Control & 29.3 & $\operatorname{secs}$ \\
\hline Monocytes & 4 & $\%$ & Total protein & 6.2 & $\mathrm{~g} / \mathrm{dl}$ & D-dimer & 57.7 & $\mu \mathrm{g} / \mathrm{ml}$ \\
\hline Lymphocytes & 5 & $\%$ & Albumin & 2.3 & $\mathrm{~g} / \mathrm{d} \mathrm{l}$ & Antithrombin III & 44.1 & $\%$ \\
\hline Red-cell count & $271 \times 10^{4}$ & $/ \mu 1$ & Total bilirubin & 2.4 & $\mathrm{mg} / \mathrm{dl}$ & Fibrinogen & 300 & $\mathrm{mg} / \mathrm{dl}$ \\
\hline Hemoglobin & 9.1 & $\mathrm{~g} / \mathrm{dl}$ & Conjugated bilirubin & 1.1 & $\mathrm{mg} / \mathrm{dl}$ & & & \\
\hline Hematocrit & 27.4 & $\%$ & Aspartate aminotransferase & 75 & IU/L & \multicolumn{3}{|c|}{ [Blood gas analysis (under $10 \mathrm{~L} / \mathrm{min} \mathrm{O}_{2}$ ] } \\
\hline Platelet count & $15.3 \times 10^{4}$ & $/ \mu 1$ & Alanine aminotransferase & 45 & $\mathrm{IU} / \mathrm{L}$ & $\mathrm{pH}$ & 7.48 & \\
\hline [Infection] & & & $\gamma$-glutamyl transpeptidase & 98 & $\mathrm{IU} / \mathrm{L}$ & $\mathrm{PaO}_{2}$ & 137 & $\mathrm{mmHg}$ \\
\hline 1,3 - $\beta$-D-glucan & 4.9 & $\mathrm{pg} / \mathrm{ml}$ & Lactate dehydrogenase & 350 & $\mathrm{IU} / \mathrm{L}$ & $\mathrm{PaCO}_{2}$ & 31 & $\mathrm{mmHg}$ \\
\hline HBs antigen & negative & & Alkaline phosphatase & 559 & $\mathrm{IU} / \mathrm{L}$ & $\mathrm{HCO}_{3}^{-}$ & 25 & $\mathrm{mmol} / \mathrm{L}$ \\
\hline HCV antibody & negative & & Total cholesterol & 71 & $\mathrm{mg} / \mathrm{dl}$ & Base excess & -0.5 & $\mathrm{mmol} / \mathrm{L}$ \\
\hline HIV antibody & negative & & Cholinesterase & 37 & IU/L & & & \\
\hline
\end{tabular}

HCV, hepatitis C virus; HIV, human immunodeficiency virus

protein and D-dimer levels were high. His chest radiograph showed a large cardio-thoracic ratio. The chest and abdominal CT scans revealed the findings of cardiogenic pulmonary edema and liver cirrhosis with ascites. The cultures of the pus on the left shoulder and the sputum but not of the urine and blood showed the growth of MRSA. The electrocardiogram showed no abnormal findings but the echocardiography showed $40 \%$ of ejection fraction and normal wall motion.

He was treated with $7 \mathrm{cmH}_{2} \mathrm{O}$ of continuous positive airway pressure (CPAP) by the noninvasive positive pressure ventilator (BiPAP Vision ${ }^{\mathrm{TM}}$, Respironics, Murrysville, PA, U. S.A.). The fraction of inhaled oxygen $\left(\mathrm{FIO}_{2}\right)$ was 0.4 . He was diagnosed as having the following: 1) sepsis due to suppurative omarthritis, 2) cardiogenic pulmonary edema, 3) end-stage liver cirrhosis, 4) disseminated intravascular coagulation (DIC). An antibiotic regimen of intravenous vancomycin (VCM) and meropenem (MEPM) was started at 0.5 g twice per day (Fig. 1). On Day 4, division of laboratory reported that Candida sp., similar to Candida albicans, was detected in the sputum culture from Day 2. An antifungal agent was not administered because of negative serum $\beta$-Dglucan test and a single positive site of Candida spp. Furthermore, MRSA was considered pathogenic. Prosthesis evulsion operation was performed and he was retuned to the ICU on the invasive ventilator on Day 4. The ventilator setting was volume controlled, $\mathrm{FIO}_{2} 1.0$, and positive endexpiratory pressure (PEEP) $7 \mathrm{cmH}_{2} \mathrm{O}$. The ABG analysis revealed; $\mathrm{PaO}_{2} 135 \mathrm{mmHg}, \mathrm{PaCO}_{2} 43 \mathrm{mmHg}, \mathrm{pH}$ 7.38. He developed acute respiratory distress syndrome (ARDS) and was treated with steroid pulse therapy (methylprednisolone 1 $\mathrm{g}$ daily for 3 days). C. dubliniensis was isolated from the aspirated sputum of Day 2 and Day 7. Selective digestive decontamination (SDD) had been performed using $0.5 \mathrm{~g}$ of VCM, $100 \mathrm{mg}$ of amphotericin B (AMPH-B) and 1000,000 units of polymyxin B twice per day since Day 9. Although the trough and peak concentrations of VCM were 14.9 and $25.6 \mu \mathrm{g} / \mathrm{ml}$, respectively, the sputum cultures still remained positive for MRSA but not for C. dubliniensis. $\beta$-D-glucan tests were all negative on Day 4 and Day 18. He was extubated on Day 10 and discharged to the general ward on Day 17.

\section{Microbiology Results}

Broth microdilution minimal inhibitory concentrations (MICs) were determined according to Clinical and Laboratory Standards Institute (formerly National Committee for Clinical Laboratory Standards) document M27-A guidelines (ASTY $^{\mathrm{TM}}$, Kyokuto, Tokyo, Japan). MICs of AMPH-B, flucytosine (5-FC), FLCZ, itraconazole (ITCZ), miconazole (MCZ) and micafungin (MCFG) were $0.25, \leq 0.125,0.25$, $0.25,0.125$ and $2 \mu \mathrm{g} / \mathrm{ml}$, respectively.

The automated method for the identification of yeasts (API ID $32 \mathrm{C}^{\mathrm{TM}}$, bioMériux, Lyon, France) showed 99.9\% identification for $C$. dubliniensis after 48 hours incubation. The identification was confirmed by the nested polymerase chain reaction (PCR), using primer mixes of Candida DNA topoisomerase II genes (5). Furthermore, it was reconfirmed to be germ tube positive and to produce abundant chlamydospores on cornmeal agars (Fig. 2).

\section{Discussion}

This is the first report describing $C$. dubliniensis isolated from the sputum of a HIV-negative patient in Japan as far as we know. The incidence of infection or colonization due to C. dubliniensis is not known in Japan, largely because of the difficulty in distinguishing this species from the morphologically similar C. albicans. First, this Candida sp. was also misidentified as $C$. albicans by green colony color on CHROMagar $^{\mathrm{TM}}$ Candida medium at our division of laboratory. However, the susceptibility of the Candida sp. against MCFG was different from that of C. albicans and they performed a further examination of a sugar assimilation test with the API ID $32 \mathrm{C}^{\mathrm{TM}}$, which showed $99.9 \%$ identification 


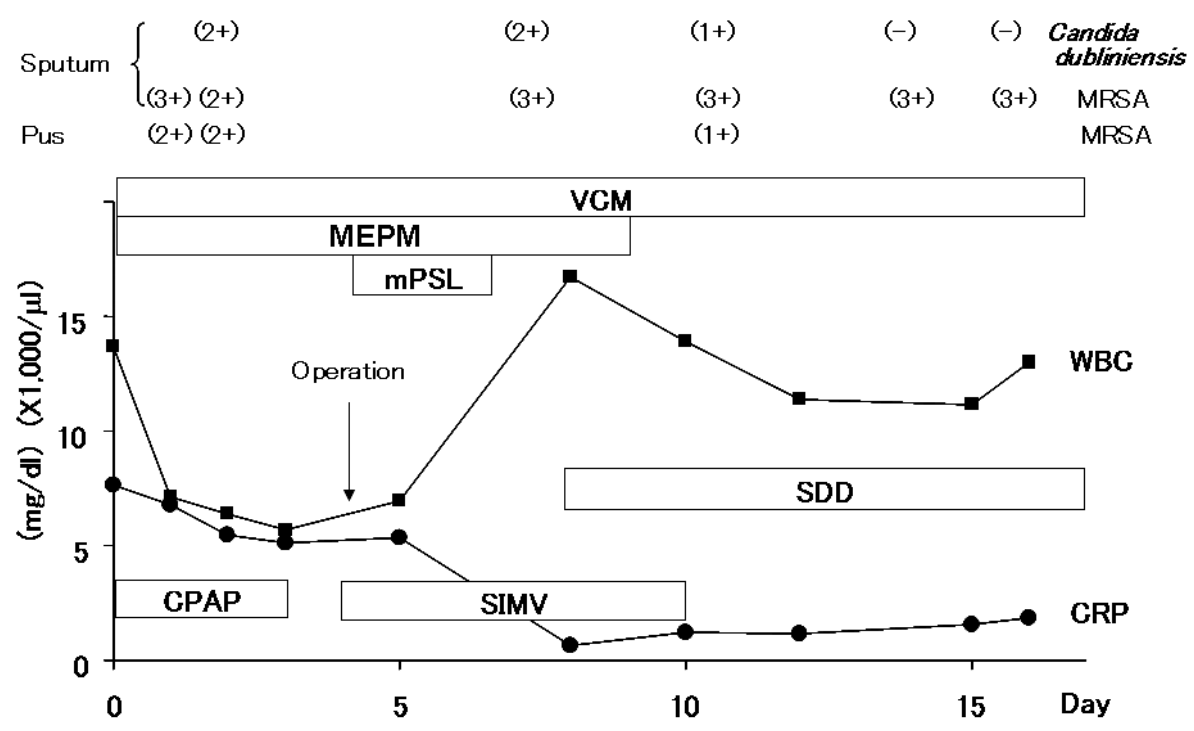

Figure 1. Clinical course of the patient. VCM, vancomycin; MEPM, meropenem; mPSL, methylprednisolone; SDD, selective digestive decontamination; CPAP, continuous positive airway pressure; SIMV, synchronized intermittent mandatory ventilation.
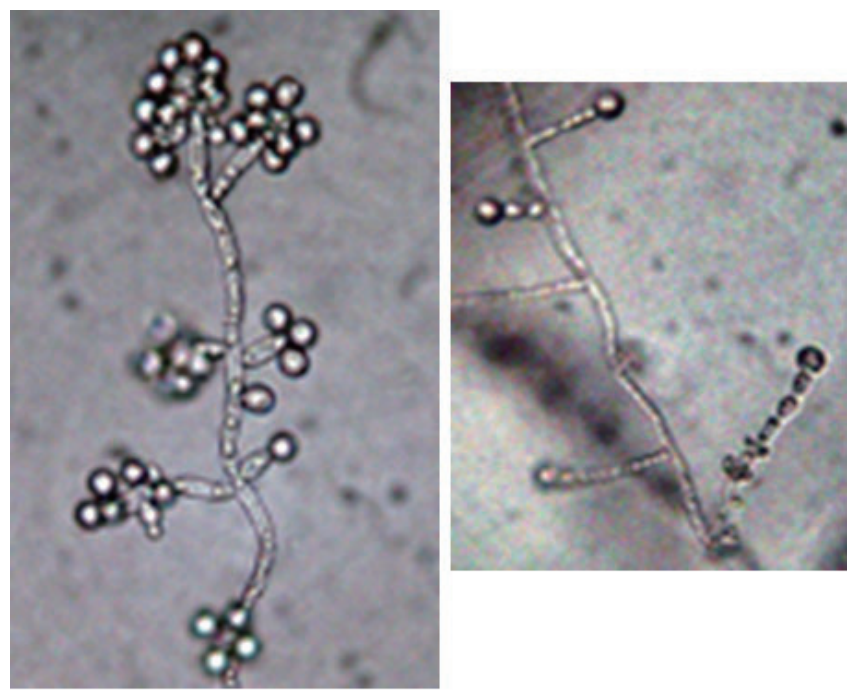

C. dubliniensis

C. albicans

Figure 2. Chlamydospore productions of Candida dubliniensis and Candida albicans on cornmeal agar plates incubated for 4 days. The production of abundant chlamydospores by $C$. dubliniensis was found $(\times 400)$.

for C. dubliniensis. Therefore, it resulted in the isolation of C. dubliniensis.

C. dubliniensis, first isolated from oropharyngeal lesions of AIDS patients in Dublin, Ireland in 1995 (6), and has a worldwide distribution (7). C. dubliniensis has been recovered from not only HIV-infected individuals with/without oral candidiasis but also HIV-negative healthy individuals without oral candidiasis (8). C. dubliniensis isolates were recovered from the blood, urine, sputum, tracheal aspirates, high vaginal swabs and so on in HIV-negative patients (2).
The two patients, in whom C. dubliniensis was recovered from the blood cultures, had end-stage liver disease (9). They speculated that the gastrointestinal (GI) tract was the source of the $C$. dubliniensis in these patients. The present patient had not been treated with antifungal agents and had end-stage alcoholic liver cirrhosis. C. albicans and other less pathogenic species such as $C$. glabrata, $C$. krusei and $C$. tropicalis were not identified but MRSA was isolated. The relationship between $C$. dubliniensis and MRSA, and interaction between anti-MRSA agents and environmental conditions for $C$. dubliniensis were not known. However, we suggested that the source of $C$. dubliniensis was the GI tract or oral cavity. The reason for positive $C$. dubliniensis from the sputum was considered to be that it had been aspirated from the GI tract or oral cavity.

Suppurative omarthritis due to MRSA and colonization of MRSA and $C$. dubliniensis in the respiratory tract were finally diagnosed for the infection in this patient. Accordingly, he was treated using SDD for $C$. dubliniensis colonization according to Japanese "guidelines for the diagnosis and treatment of deep-seated mycosis" (10). There were several risk factors for invasive candidiasis, including elderly ( $\geq$ 70), prolonged use of broad spectrum antimicrobials (more than 2 classes, $\geq 7$ days), total parenteral nutrition, severely malnourished, use of H2-blockers, prolonged mechanical ventilation ( $\geq 48 \mathrm{hrs)}$ and intravascular catheterization, urinary catheterization ( $\geq 1$ week). Although mortality benefit of SDD for the patients undergoing liver transplantation has not been shown, it has been shown to decrease the number of infections (11). Actually, C. dubliniensis was not recovered 7 days after the initiation of SDD and it was not identified from the other sites. Not only SDD but also supportive care such as nutrition might be effective for its elimination.

The clue to identify $C$. dubliniensis was the incomprehen- 
sible MIC of MCFG $(2 \mu \mathrm{g} / \mathrm{ml})$ if it had been C. albicans. Pfaller et al reported the validation of in vitro methods for determining the susceptibilities of Candida spp. to caspofungin (CSP), one of the echinocandin-class antifungal agents (12). They showed the usefulness of RPMI medium, MIC endpoint criterion of partial inhibition and incubation for 24 hours. Consequently, they revealed $0.25 \mu \mathrm{g} / \mathrm{ml}$ of MIC at which $90 \%$ of $C$. dubliniensis isolates are inhibited
$\left(\mathrm{MIC}_{90}\right.$ ) against CSP (13). When we reevaluated the MIC of MCFG by their methods, the MIC was decreased to 0.016 $\mu \mathrm{g} / \mathrm{ml}$. Taken together, encountering the puzzling susceptibility of C. albicans against MCFG, it may be necessary to discriminate it from $C$. dubliniesis by use of API ID $32 \mathrm{C}^{\mathrm{TM}}$. The present case emphasizes the need to recognize the new Candida sp., C. dubliniesis when encountering immunocompromised patients.

\section{References}

1. Vazquez JA, Dembry LM, Sanchez V, et al. Nosocomial Candida glabrata colonization: an epidemiologic study. J Clin Microbiol 36: 421-426, 1998.

2. Fotedar R, Al-Hedaithy SS. Candida dubliniensis at a university hospital in Saudi Arabia. J Clin Microbiol 41: 1907-1911, 2003.

3. Jabra-Rizk MA, Baqui AAMA, Kelley JI, Falkler WA Jr, Merz WG, Meiller TF. Identification of Candida dubliniensis in a prospective study of patients in the United States. J Clin Microbiol 37: 321-326, 1999.

4. Moran GP, Sullivan DJ, Henman MC, et al. Antifungal drug susceptibilities of oral Candida dubuliniensis isolates from human immunodeficiency virus (HIV)-infected and non-HIV-infected subjects and generation of stable fluconazole-resistant derivatives in vitro. Antimicrob Agents Chemother 41: 617-623, 1997.

5. Kanbe T, Horii T, Arishima T, Ozeki M, Kikuchi A. PCR-based identification of pathogenic Candida species using primer mixes specific to Candida DNA topoisomerase II genes. Yeast 19: 973989, 2002.

6. Sullivan DJ, Westerneng TJ, Haynes KA, Bennett DE, Coleman DC. Candida dubliniensis sp. nov.: phenotypic and molecular characterization of a novel species association with oral candidosis in HIV-infected individuals. Microbiology 141: 1507-1521, 1995.
7. Sullivan D, Coleman D. Candida dubliniensis: characteristics and identification. J Clin Microbiol 36: 329-334, 1998.

8. Coleman DC, Sullivan DJ, Bennett DE, Moran GP, Barry HJ, Shanley DB. Candidiasis: the emergence of a novel species, Candida dubliniensis. AIDS 11: 557-567, 1997.

9. Brandt ME, Harrison LH, Pass M, et al. Candida dubliniensis fungemia: the first four cases in North America. Emerg Infect Dis 6: 46-49, 2000.

10. Deep-seated mycosis guidelines editorial committee: Guidelines for the diagnosis and treatment of deep-seated mycosis. Ishiyaku, Tokyo, 2003: 31-33 (in Japanese).

11. Ong J, Younossi ZM. Selective digestive decontamination and its use in patients with liver disease. Disease Management and Health Outcomes 4: 17-26, 1998.

12. Pfaller MA, Messer SA, Boyken L, et al. Further standardization of broth microdilution methodology for in vitro susceptibility testing of caspofungin against Candida species by use of an international collection of more than 3,000 clinical isolates. J Clin Microbiol 42: 3117-3119, 2004.

13. Pfaller MA, Diekema DJ. Rare and emerging opportunistic fungal pathogenesis: concern for resistance beyond Candida albicans and Aspergillus fumigatus. J Clin Microbiol 42: 4419-4431, 2004.

(C) 2007 The Japanese Society of Internal Medicine http://www.naika.or.jp/imindex.html 